\title{
GLORIA and Study of Cataclysmic Variables
}

\author{
R. Hudec ${ }^{1,2}$, V. Šimon ${ }^{1,2}$ \\ ${ }^{1}$ Astronomical Institute, Academy of Sciences of the Czech Republic, CZ-25165 Ondřejov, Czech Republic \\ ${ }^{2}$ Czech Technical University in Prague, Faculty of Electrical Engineering, Prague, Czech Republic
}

Corresponding author: rene.hudec@asu.cas.cz

\begin{abstract}
We report here on the ongoing EU FP7 Project GLORIA (GLObal Robotic-telescopes Intelligent Array) with emphasis on possibility of investigation of cataclysmic variables by users. GLORIA will enable the first free and open-access network of robotic telescopes in the world. We show several examples of the not often used topics (but suitable for GLORIA) for the studies of activity of cataclysmic variables, e.g. search for outbursts in intermediate polars and the fluctuations of brightness in their quiescence, and investigation of the optical counterparts of supersoft X-ray sources.
\end{abstract}

Keywords: catalysmic variables: optical photometry - spectroscopy: low-dispersion spectra - data mining - robotic telescopes - project EU FP7 GLORIA.

\section{Introduction}

The research in astronomy poses two main challenges, namely 1) the immensity of the sky and 2) the huge amount of astronomical data being gathered. In fact, astronomers are nowadays facing great difficulty in finding the resources to analyze the increasing flood that modern astronomy instruments generate,insufficient computing power, insufficiently powerful software tools, and not enough hours in the day. The sky comprises 40000 square degrees (144 million square minutes). The future professional projects (like LSST) intend to observe a very significant fraction of it on a regular basis. In order to meet the abovementioned challenges, an increasing number of astronomical projects have begun to try to foster citizen participation to help analyze data by using collaborative Internet application (the so-called Web 2.0).

GLORIA stands for "GLObal Robotic-telescopes Intelligent Array". GLORIA will be the first free and open-access network of robotic telescopes in the world. It represents a Web 2.0 environment where users can do research in astronomy by observing with robotic telescopes, and/or by analyzing data that other users have acquired with GLORIA, or from other free access databases like the European Virtual Observatory (http://www.euro-vo.org).

The GLORIA project (http://gloria-project.eu) represents the first attempt to create a network of about 20 robotic telescopes for public access, education, science and much much more. The GLORIA Network has 17 network telescopes now. Few others are expected to join us soon.
Many Internet communities have already formed to speed-up scientific research, to collaborate in documenting something, or for social projects. Research in astronomy can benefit from attracting many eyes to the sky - to detect something in the sky requires looking at the right place at the right time. Our robotic telescopes can search the sky, but the vast quantities of data they produce are far greater than astronomers have time to analyze. GLORIA will provide a way of putting thousands eyes and minds on an astronomy problem. GLORIA is intended to be a Web 2.0 structure, with the possibility of doing real experiments. The community will not only generate content, as in most Web 2.0, but it will control telescopes around the world, both directly and via scheduled observations. The community will take decisions for the network and it will give "intelligence" to GLORIA, while the drudge work (such as drawing up telescope schedules that satisfy various constraints) will be done by algorithms that will be developed for the purpose.

GLORIA project will define free standards, protocols and methodology for the following purposes: controlling Robotic Telescopes and all related instrumentation (i.e. cameras, filter-wheels, domes...); giving Web access to the Network: access to an arbitrary number of robotic telescopes via a web portal; conducting Online experiments (it will be possible to design specific web environments for controlling telescopes for research on a specific scientific issue; conducting Off-line experiments (it will be possible to design specific web environments for analyzing astronomical meta-data produced by GLORIA or other databases). 
The main part of GLORIA is represented by providing access to robotic telescopes, Online experiments (observations), Offline experiments (research on acquired images), Live broadcasting, Social network + community, and Support.

\section{Objectives and Benefits of GLORIA Project}

The world-wide network of robotic telescopes (GLORIA) does not intend to compete with LSST, but our underlying idea is that "the more eyes we put on the sky, the more and greater the scientific discoveries will be achieved". Thus, in order to try to improve the way we are doing astronomy research, this project aims to build the first open access world wide network of robotic telescopes to serve citizens from around the world for free, but competing for observing time. Hence GLORIA is indeed an "Intelligent Array" and it bases its intelligence in its community of users (Sanchez Moreno et al. 2013). Like most Web 2.0 projects, GLORIA implements a reputation-based scoring system to reward user contributions, driven by parameters such as the quality of the gathered and processed images, number of hours invested in the observations etc., as well as the votes granted by the rest of the community that finally evaluate the quality of the work done. At the end of month 24 (October 2013) the consortium produced a standard for adding new telescopes and experiments to this network. Unlike other private, profitable ongoing initiatives, the GLORIA network will provide a free, twofold service to the community:

1. Giving citizens (including professional and amateur astronomers) free access to a network of 17 robotic telescopes spread into 4 continents and both hemispheres. In the case of professional astronomers, preferences will be given to those of developing countries who will lack astronomical facilities in their own nations. 2. Giving citizens an easy web access to all the data collected by the robotic telescopes.

Beside giving service to research, in order to manage GLORIA main objective of fostering astronomical research, by allowing near continuous observations of a given target thanks to its world-wide network facilities, the following additional tangible outcomes will be pursued:

1. GLORIA will enable more telescopes to join, producing methodology, standards, software, and documentation oriented to teach professional and amateurs astronomers to robotize their telescopes and to integrate them into the GLORIA network.

2. GLORIA will enable more research goals to be pursued, producing methodology, standards, software, and documentation oriented to teach amateurs and professional astronomers to design new web experiments and to integrate them into the GLORIA network.

3. GLORIA will encourage participation in order to increase in number its community of citizen scientists. Newcomers are very welcomed.

4. GLORIA will give free access to knowledge to everybody. All knowledge (software, manual, standards, documents, astronomical images, data, etc) produced by the GLORIA consortium and by the community will have copyleft licenses.

5. GLORIA intends to continue in the future. Since the maintenance of the distributed telescopes is paid by their owners, the cost of the core part is very cheap. The consortium believes that an economic model based in public and private subventions and donations is very possible for a project like this to go on beyond 2014 .

\subsection{Impact}

The GLORIA partners really believe in the enormous power of astronomy as a center of interest in scientific and human training of our young people. The children of today will be the astronomers of tomorrow. In order to enroll newcomers and awaken interest in astronomy among children, during 2012-14 we will organize the live Internet broadcast of 5 astronomical events: 4 eclipses and a transit of Venus, which will be made from the GLORIA network, with associated activities in all schools of the partners countries, with the aim of getting students and teachers participating in researchbased science education and improving their motivation to push the barriers of science education further.

\subsection{The GLORIA consortium}

GLORIA brings together a critical mass of scientific and technological leaders with extensible expertise in TIC and Astronomical research, dissemination and developed of technological projects. The GLORIA consortium includes these institutions: UPM-Technical University of Madrid Computer Science Faculty-Spain (project coordination); AUAV-Astronomical Institute, Academy of Sciences of the Czech Republic; CSIC-Spanish Research Council-Spain (scientific coordination); CVTUCzech Technical University-Czech Republic, IP-ASCRInstitute of Physics of the Academy of Sciences of the Czech Republic; IAC-Astrophysic Institute of Canarias-El Teide Observatory-Spain; INAF-Istituto Nazionale di Astrofisica-Italy; SAO-Special Astrophysical Observatory-Russia; UCDNUID-University College Dublin-Ireland; UC-University of ChileChile; UMA-University of Málaga-Spain; UOXFOxford University-U.K.; UNIWARSAW-University of Warsaw-Poland. 


\section{GLORIA and Cataclysmic Variables}

Cataclysmic variables (CVs) represent important targets for GLORIA observations mainly because of the unique properties of their activity. The category of these systems as a whole displays a very broad range of the optical activity observable by the common CCD photometers (e.g. flickering on the timescale of minutes and the amplitude of several tenths of magnitude, the orbital modulation sometimes with the amplitude of more than a magnitude, outbursts or transitions between the high and low states on the timescales of days or weeks and the amplitudes of several magnitudes).

The basic properties of the optical emission of CVs need to be taken into account for the optimal strategy of monitoring of these targets in citizen science mode. We will show some examples of activity of various types of CVs which can be the targets for the GLORIA observers.

\subsection{Distribution of cataclysmic variables the sky}

Generally, celestial objects are not distributed uniformly in the sky mainly because of the structure of our Galaxy.

As shown by Warner (1995), most known CVs are observed over the whole sky. The reason is that CVs represent rather low-luminosity population, hence only the nearby such objects are bright enough to be observed. This relatively isotropic distribution implies that all the observatories at any latitude, included in GLORIA, will have the opportunity to choose a sufficiently large amount of suitable CVs.

Only known classical novae concentrate toward the Galactic plane and the Galactic bulge. The reason for this is the high optical luminosity in their explosion, hence even the objects at the large distances from the Earth are observable. The direction toward the center of the Galaxy is therefore also suitable for searching for explosions of classical novae with a monitor. This favors the GLORIA observatories located in the Southern Hemisphere.

\section{$3.2 \quad$ Peak magnitudes of CVs}

The catalog of Downes et al. (2001) shows that the apparent peak magnitudes of various CV types differ from each other. The reason is that there is a significantly different typical optical luminosity of each type. The explosions of classical novae dominate in the brightest part of the distribution. Their peak magnitude is often near 9-10 (but some of them may be even brighter), so they are easily detectable even by small-aperture, wide-field monitors. The brightest known dwarf novae in outburst can reach about 10-12 mag but about
13 mag is more typical. CVs with strongly magnetized white dwarf (polars) (e.g. Warner 1995) are rather faint systems (13-20 mag), so their observing requires the largest available telescopes.

\subsection{Prospects of CV investigation by GLORIA}

Below we give some examples of $\mathrm{CV}$ types suitable for investigation within GLORIA project.

Dwarf novae with their large-amplitude (3-5 mag) outbursts with the typical recurrence time from weeks to months (e.g. Warner 1995; Hameury et al. 1998) are definitely very promising targets even for the modest telescopes included in GLORIA.

We also emphasize the need of observing the outbursts of some intermediate polars (i.e. CVs with a mildly magnetized white dwarf) (e.g. Warner 1995). These systems may be promising GLORIA targets, as illustrated by the optical activity of DO Dra/2A 1150+720 (Fig. 1). What typical features of activity of this object can be explored by GLORIA telescopes? (1) Rare strong outbursts with the amplitude of 4-5 mag, lasting for several days and separated by $\sim 800$ days. These short outbursts with the decay faster than exponential and faster than predicted for non-magnetic dwarf novae are in accordance with Angelini \& Verbunt's (1989) model for outbursts in a disk with a large missing inner region (caused by the magnetosphere of the white dwarf). (2) Strong and unpredictable fluctuations on a timescale of several days or weeks (much longer than the orbital period).

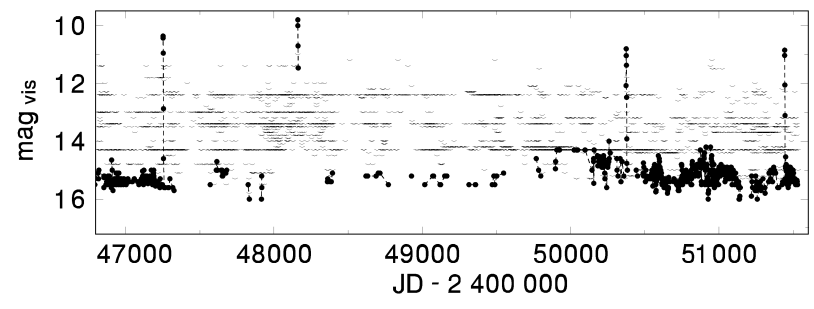

Figure 1: The light curve of the intermediate polar DO Dra with rare outbursts. The points denote the one-day means, the $v$ symbols represent the upper limits of brightness. Adapted from Šimon (2000).

The short outbursts or rather flares of uncertain origin may occur in various intermediate polars but since they are short, extensive monitoring is needed to detect them (van Amerongen \& van Paradijs 1989; Warner 1995).

Supersoft X-ray sources (van den Heuvel et al. 1992) represent another example of interesting objects to be investigated by GLORIA experiments. Since the very soft X-ray emission is easily absorbed both inside 
the source and the interstellar medium, only a small fraction of supersoft X-ray sources is observable in the $\mathrm{X}$-ray spectral region. The specific spectroscopic properties of their optical emission give a hope to discover more such objects even without detecting their X-ray emission (Steiner \& Diaz 1998). The system V Sge (Herbig et al. 1965) can be considered to be a member of this category (Steiner \& Diaz 1998; Greiner \& van Teeseling 1998). Since it is relative bright (10$13 \operatorname{mag}(V)$ ), it is easily observable by various robotic telescopes. V Sge shows a very complicated long-term optical activity. Its outbursts were observed mainly in the first half of the twentieth century (Šimon \& Mattei (1999). However, this activity changed considerably with time. A segment of the long-term activity starting in the 1960s is displayed in Fig. 2). It consists of the one-day means of brightness. The high/low state transitions occurred in some time segments. They were separated by the so-called flat segments (e.g. in the surroundings of JD 2445000 ), in which only small fluctuations of brightness remained. We can thus conclude that observing at various times captures this object in different phases of its strong activity.

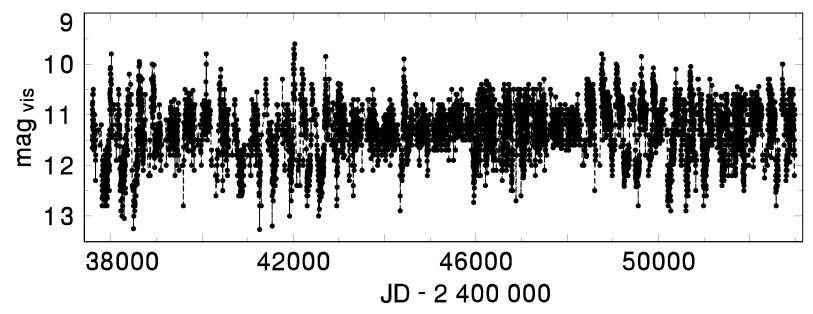

Figure 2: Long-term activity of the supersoft $\mathrm{X}$ ray source $\mathrm{V}$ Sge in the optical band (AAVSO and AFOEV data). Notice the large variations of activity on the timescale of several years (from the segments with only small fluctuations to the segments with the large-amplitude transitions between the high and low states). Adapted from Šimon \& Mattei (1999).

Dividing the series of the optical (CCD and photoelectric) observations of V Sge, obtained at different nights (Fig. 3), into the groups according to the states of the long-term activity enables to obtain the profile of the typical orbital modulation (the orbital ephemeris of Smak 1995 was used). This figure shows the intensity transfered from magnitudes. Although the profile of the orbital modulation can be resolved in the individual night series, the orbital period of 12.34 hours (Smak 1995) does not enable to observe the whole period from a given observing site, so a multisite campaign is desirable, especially because the orbital modulation and the long-term variations of brightness are superimposed. This means that observations obtained by GLORIA may provide valuable information. When the intensity of a given nigh series belonging to a given such group is shifted to match a template, it is possible to obtain the mean profile of the orbital modulation characteristic for the group (Fig. 3).

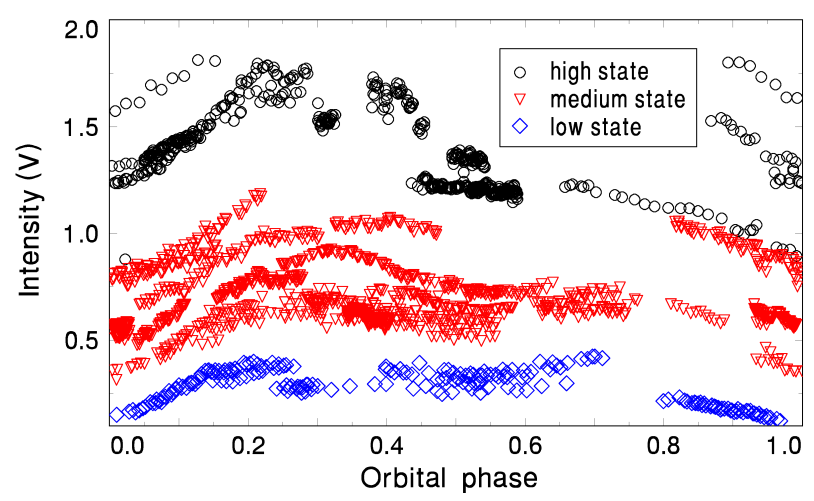

Figure 3: Photoelectric and CCD observations of V Sge in the intensity scale, folded with the orbital phase (the orbital ephemeris of Smak 1995). The sorting into three groups is marked. Adapted from Šimon et al. (2002).

The CV in Pegasus (found in eruption in May 8, 2010) can serve as an excellent example of a bright object which can be easily detected by amateurs and small cameras and/or telescopes. This object represented a rare type of dwarf nova with very long recurrence time (67 years) but with very large amplitudes (Hudec 2010). We note that the second flare of this CV in 1942 was found using astronomical photographic archives which are perfectly suitable for this kind of work, i.e. searches for past historical flare events (Hudec 1999 and 2007, Hudec et al. 2012). Only very few such objects are known because they can easily escape detection.

In this regard, we also note that the sky contains many yet unknown transients of various kinds. They are identified and attract attention only when they show themselves in their outbursts. The follow-up observations then can lead to the study of their type. One such object detected by a very small telescope is the Liška Flare Star. It is an example of the astronomical discovery of a very rare event with a small amateur telescope (Liška et al. 2014). We suggest that this object is a new flare dMe star of UV Ceti type based on its color, X-ray emission, and the properties of the optical light curve of the flare, identical with the X-ray sources RX J1118.3+1347 and 2E 1115.8+1403.In SIM$\mathrm{BAD}$, these 2 sources are given as different sources but this is probably wrong and it is only one source. The object may be similar to the EXOSAT X-ray source EXO $020528+1454.8=$ G $035-027$ with a dMe flare star counterpart detected by Hudec et al. (1988). 


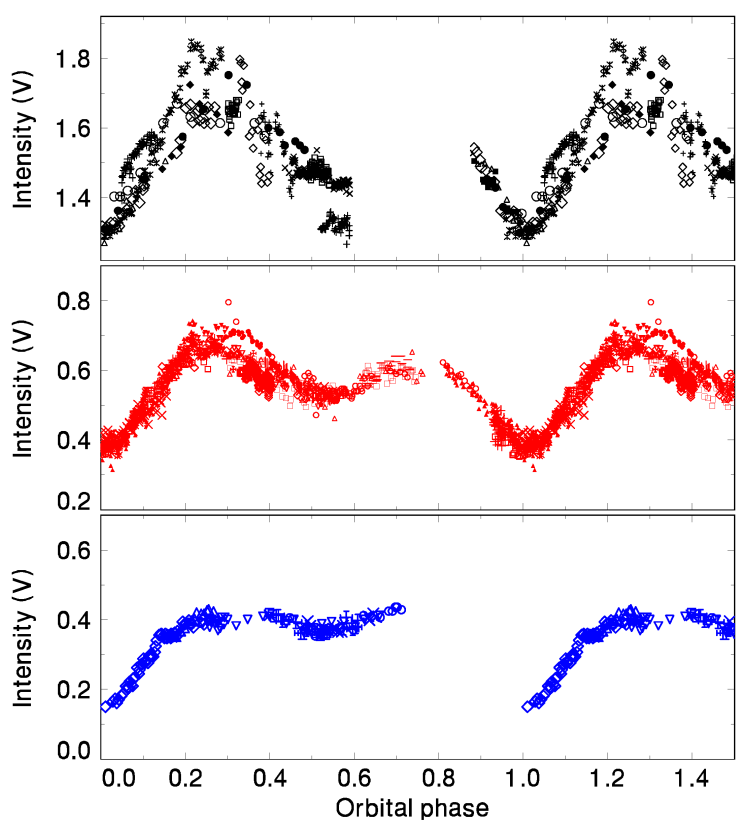

Figure 4: Photoelectric and CCD observations of V Sge from Fig. 3, now sorted into three groups according to their mean intensity (the orbital ephemeris of Smak 1995). The individual curves of each group were shifted in intensity to match the template. The scale of the ordinate is identical for all three plots. Adapted from Šimon et al. (2002).

The observations and research of CVs are expected to be supervised by GLORIA scientists. Everybody can join us at http://gloria-project.eu, and this involves both citizen science and students, as well as professional astronomers. Typically 20 percent of Robotic Telescopes (RTs) observing time is expected to be used for GLORIA users, but some telescopes such as BART will provide up to 40 percent.

\section{Acknowledgement}

GLORIA is project supported by the EU FP7 program (No. 283783). The scientific part of the study is linked to the the GA CR grant 13-39464J (optical analyses) while the long-term $\mathrm{X}$-ray analyses are related to GA CR grant 113-33324S. We used the observations from the AAVSO International database (Massachusetts, USA) and the AFOEV database operated in Strasbourg, France. We thank the variable star observers worldwide whose observations contributed to this analysis as well.

\section{References}

[1] Angelini, L., Verbunt, F., 1989, MNRAS, 238, 697 doi:10.1093/mnras $/ 238.3 .697$
[2] Downes, R. A., et al., 2001, PASP, 113, 764 doi:10.1086/320802

[3] Greiner, J., van Teeseling, A., 1998, A\&A, 339, L21

[4] Hameury, J.-M., et al., 1998, MNRAS, 298, 1048 doi:10.1111/j.1365-8711.1998.01773.x

[5] Herbig, G. H., et al., 1965, ApJ, 141, 617 doi: $10.1086 / 148149$

[6] Hudec, R., 2010, The Astronomer's Telegram, No. 2619

[7] Hudec, R., et al., 1988, Astronomical Institutes of Czechoslovakia, Bulletin (ISSN 0004-6248), vol. 39 , no. 5 , p. 296-302

[8] Hudec, R., et al., 2012, Acta Polytechnica, IBWS2011 Proceedings, 1(52)

[9] Hudec, R., 2007, in Exploring the Cosmic Frontier: Astrophysical Instruments for the 21st Century. ESO Astrophysics Symposia, ISBN 978-3-540-39755-7. Germany, p.79 doi:10.1007/978-3-540-39756-4_25

[10] Hudec, R., 1999, An introduction to the world's large plate archives, Acta Historica Astronomiae, vol.6, 28

[11] Liška, J., et al., 2014, Acta Polytechnica, accepted

[12] Sanchez Moreno, F. M., et al., 2013, GLORORA documentation package

[13] Šimon, V., Mattei, J. A., 1999, A\&AS, 139, 75

[14] Šimon, V., 2000, A\&A, 360, 627

[15] Šimon, V., Hric, L., Petrík, K., et al., 2002, A\&A, 393, 921

[16] Smak, J., 1995, Acta Astron., Vol. 45, 361

[17] Steiner, J. E., Diaz, M. P., 1998, PASP, 110, 276 doi:10.1086/316139

[18] van Amerongen, S., van Paradijs, J., 1989, A\&A, 219,195

[19] van den Heuvel, E. P. J., et al., 1992, A\&A, 262, 97

[20] Warner, B., 1995, Cataclysmic Variable Stars, Cambridge Univ. Press, Cambridge doi:10.1017/CB09780511586491 